OPEN

\author{
SUBJECT AREAS:
}

MAGNETIC PROPERTIES

AND MATERIALS

APPLIED PHYSICS

CHEMICAL PHYSICS

FERROELECTRICS AND

MULTIFERROICS

Received

12 June 2013

Accepted

23 September 2013

Published

9 October 2013

Correspondence and requests for materials should be addressed to X.M.L. (xiaomeil@nju. edu.cn)

\title{
Peculiar magnetism of $\mathrm{BiFeO}_{3}$
}

\section{nanoparticles with size approaching the period of the spiral spin structure}

\author{
Fengzhen Huang' 'Zhijun Wang', Xiaomei Lu', Junting Zhang' ', Kangli Min', Weiwei Lin², Ruixia Ti', \\ TingTing $\mathrm{Xu}^{\prime}$, Ju He', Chen Yue' \& Jinsong Zhu'
}

\footnotetext{
'National Laboratory of Solid State Microstructures, Physics School, Nanjing University, Nanjing 210093, People's Republic of China, ${ }^{2}$ Institut d'Electronique Fondamentale, Université Paris-Sud, Orsay 91405 , France.
}

Size effect of multiferroics is important for its potential applications in new type miniaturized multifunctional devices and thus has been widely studied. However, is there special size effect in the materials with spiral modulated spin structure (such as $\mathrm{BiFeO}_{3}$ )? It is still an issue to be investigated. In this report, structural, magnetic and magnetoelectric coupling properties are investigated for sol-gel prepared $\mathrm{BiFeO}_{3}$ nanoparticles with various sizes. It is found that a structural anomaly arises for the particles with size close to the $62 \mathrm{~nm}$ period of the spiral modulated spin structure, which induces an obviously increased ferromagnetism. In addition, large magnetoelectric coupling effect is observed in $62 \mathrm{~nm} \mathrm{BiFeO}$ nanoparticles. Our result provides another insight into the size effect of $\mathrm{BiFeO}_{3}$, and also a clue to the magnetic structure at nanoscale.

M ultiferroics are promising materials for the design and synthesis of multifunctional devices ${ }^{1-4}$. They are noteworthy for their unique and strong coupling of electric, magnetic, and structural order parameters, giving rise to simultaneous (anti)ferroelectricity, (anti)ferromagnetism, and/or ferroelasticity ${ }^{5,6}$. As one of the most important single-phase multiferroic materials, $\mathrm{BiFeO}_{3}(\mathrm{BFO})$ has attracted much attention because it has both high ferroelectric Curie temperature of about $1103 \mathrm{~K}$ and antiferromagnetic Néel temperature of about $643 \mathrm{~K}^{7}$. The magnetic structure of BFO is of G-type antiferromagnetic, and the combined action of exchange and spin-orbit interactions produces spin canting away from perfect antiferromagnetic ordering, resulting in a spiral spin arrangement with a wavelength of about $62 \mathrm{~nm}$, and thereby producing a helimagnetic order and a vanishing magnetization in the bulk ${ }^{8,9}$. Like many other materials, $\mathrm{BFO}$ shows a variation of properties with the decrease of size ${ }^{10,11}$. Néel and Curie temperature have been shown to decrease with decreasing particle size ${ }^{12,13}$. Enhanced magnetization has been reported in nanoparticles, nanowires and nanopowders, which is thought to originate from the uncompensated spins at surface ${ }^{14-17}$, an effect that is well known in classic antiferromagnets such as $\mathrm{NiO}^{18}$. Moreover, some other distinct phenomena in $\mathrm{BFO}$ are also associated with size effect. For instance, some researchers report the fading away of the magnetic anomaly at about $50 \mathrm{~K}$ with the decrease of size, while some others provide just the opposite observation $\mathrm{s}^{15,19,20}$. Correspondingly, the physical mechanism for this magnetic anomaly is still an controversial issue at present ${ }^{15,19-22}$. In our earlier work, an interesting film thickness dependent magnetization oscillation with a period of about $62 \mathrm{~nm}$ has been observed ${ }^{23}$.

All these results suggest the exceptional and complex properties of BFO in nanoscale, and the special magnetic fluctuating behavior observed in our BFO films reminds us of the important correlations among crystal structure, macroscopic magnetic property and the unique spiral spin structure. However, in spite of intense studies about BFO, it is still unknown what will happen when the sample size is close to the spiral spin period of BFO. Thus, in this paper, we systematically investigate the magnetic properties of BFO particles with size from $18 \mathrm{~nm}$ to $248 \mathrm{~nm}$. Similar to other reports, it is observed that the ferromagnetic property basically increases with decreasing particle size because of the increased surface to volume ratio. However, our most important result is that BFO particles with size close to the spiral spin period $62 \mathrm{~nm}$ possess exceptionally enhanced ferromagnetic property and magnetodielectric effect compared with others, which is considered to be closely related to the structural anomaly at this particle size, and is also possibly associated with its intrinsic spiral-modulated spin order. 


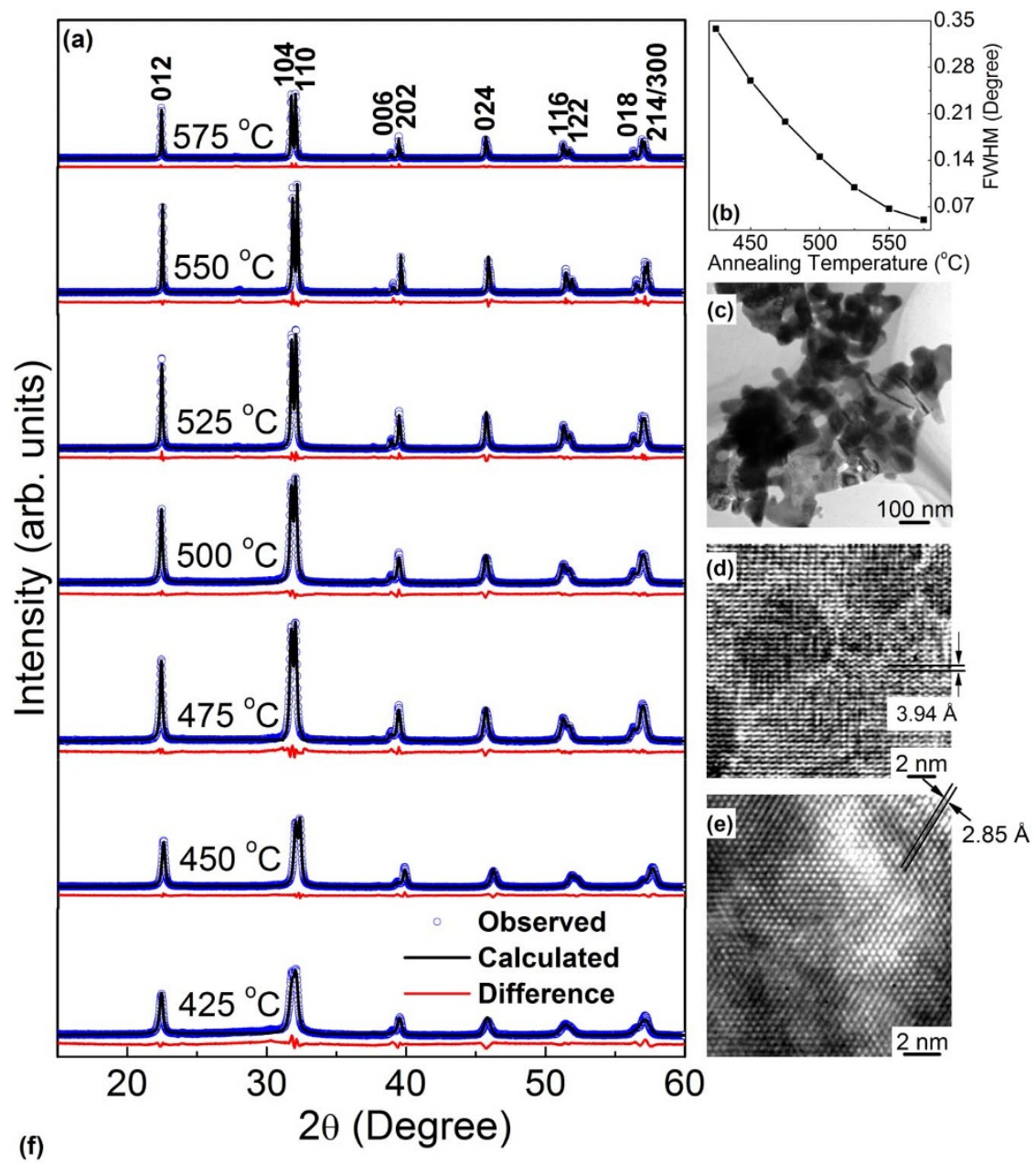

\begin{tabular}{|c|c|c|c|c|c|c|c|}
\hline$d$ & $18 \mathrm{~nm}$ & $31 \mathrm{~nm}$ & $45 \mathrm{~nm}$ & $62 \mathrm{~nm}$ & $83 \mathrm{~nm}$ & $170 \mathrm{~nm}$ & $248 \mathrm{~nm}$ \\
\hline$R_{\mathrm{p}}(\%)$ & 6.22 & 2.36 & 4.26 & 2.03 & 3.56 & 4.95 & 3.70 \\
\hline$R_{w p}(\%)$ & 7.89 & 3.06 & 5.91 & 2.80 & 4.66 & 6.77 & 5.04 \\
\hline $\mathrm{a}(\AA)$ & 5.573 & 5.561 & 5.578 & 5.578 & 5.579 & 5.572 & 5.578 \\
\hline $\mathrm{c}(\AA)$ & 13.838 & 13.819 & 13.868 & 13.871 & 13.870 & 13.852 & 13.869 \\
\hline
\end{tabular}

Figure $1 \mid$ (a) X-ray diffraction patterns of BFO nanoparticles annealed at various temperatures. (b) Annealing temperature dependent full width at half maximum (FWHM) of (012) peak. (c) The TEM image and (d-e) high resolution TEM images of the 62 nm BFO particles. (f) Parameters obtained from Rietveld refinement.

\section{Results}

Figure 1(a) shows the X-ray diffraction (XRD) patterns of BFO nanoparticles annealed at different temperatures, where the peaks are indexed based on Rhombohedral structure (JCPDS File No. 712494). It is seen that all the nanoparticles present single-phase perovskite structure and no obvious impurity phase is observed within the resolution of XRD. Fig. 1(b) and supplementary Fig. S1 show that the full width at half maximum of (012) peak decreases as the annealing temperature increases from 425 to $575^{\circ} \mathrm{C}$, indicating the increase of particle size. The average particle sizes (i.e. diameter of the particle, d) determined from scanning electron microscopy (SEM) (Supplementary Figs. S1-S3 and Table S1) are around $18 \pm 5 \mathrm{~nm}, 31 \pm$ $7 \mathrm{~nm}, 45 \pm 7 \mathrm{~nm}, 62 \pm 12 \mathrm{~nm}, 83 \pm 20 \mathrm{~nm}, 170 \pm 45 \mathrm{~nm}, 248 \pm$ $100 \mathrm{~nm}$ for the BFO nanoparticles annealed at $425^{\circ} \mathrm{C}, 450^{\circ} \mathrm{C}, 475^{\circ} \mathrm{C}$, $500^{\circ} \mathrm{C}, 525^{\circ} \mathrm{C}, 550^{\circ} \mathrm{C}$, and $575^{\circ} \mathrm{C}$, respectively. Typical TEM images and high resolution TEM images obtained from a portion of an individual $62 \mathrm{~nm}$ (Fig. 1 (c)-(e)) and $18 \mathrm{~nm}$ (Supplementary Fig. S4) particle further confirm the highly crystalline nature of our BFO nanoparticles. Size-dependent crystal structure of BFO nanoparticles is analyzed by Rietveld refinement based on hexagonal representation of space group $\mathrm{R} 3 \mathrm{c}$, and the results are summarized in Fig. 1 (f), where a and $c$ are the lattice parameters in hexagonal representation. No impurity phase is found in the refinement process, and the small values of $R_{w p}$ and $R_{p}$ indicate that all the BFO nanoparticles fit very well to the Rhombohedral structure.

Figure 2 (a) shows the initial magnetization curves measured at room temperature. Nonlinear curve is only observed for the $62 \mathrm{~nm}$ BFO particles at the magnetic field less than $5 \mathrm{kOe}$, indicating its different magnetic structure compared with that of all the others, since the shape of initial magnetization curve is controlled by domain structure $^{24}$. This is further confirmed by magnetization-field hysteresis measurements. Typical antiferromagnetic response similar to bulk BFO is obtained for the particles with diameters of about $170 \mathrm{~nm}$ and $248 \mathrm{~nm}$ (Supplementary Fig. S5), while ferromagnetic hysteresis loops are observed for the particles with diameter smaller than $83 \mathrm{~nm}$ (Fig. 2(b)). Moreover, as shown in the inset of Fig. 2 (b), except the abnormal point for the $62 \mathrm{~nm}$ particles, the magnetization measured at $7 \mathrm{~T}$ increases with the decreasing particle size and presents almost a linear dependence on $1 / \mathrm{d}$ (or the surface to volume ratio), indicating that these BFO nanoparticles can be modeled by a 

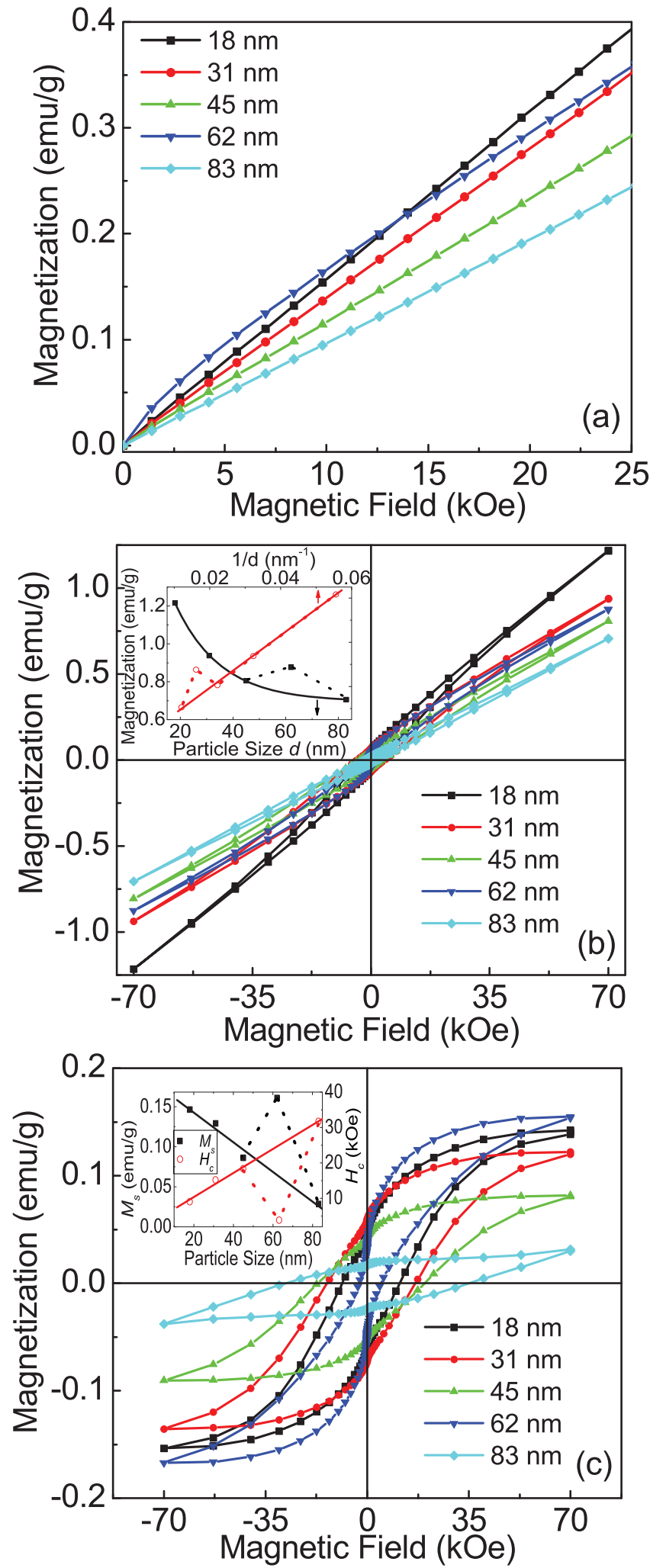

Figure $2 \mid$ (a) Initial magnetization curves, (b) room temperature magnetic hysteresis loops, (c) the ferromagnetic parts in the magnetic hysteresis curves of BFO nanoparticles with various sizes. The inset in (b) gives the magnetization at $7 \mathrm{~T}$ as a function of $\mathrm{d}$ and $1 / \mathrm{d}$, and in (c) shows size-dependent $M_{s}$ and $H_{c}$.

superposition of an antiferromagnetic core and a ferromagnetic surface, and their magnetization are mainly contributed by the uncompensated spin at grain surface ${ }^{15,18}$. The large deviation from the expected behavior for $62 \mathrm{~nm}$ BFO particles reveals the presence of new contribution to its ferromagnetism in addition to the uncompensated spin at the grain surfaces. In order to get a clearer image of ferromagnetic contribution in the hysteresis curves, the linear contributions from antiferromagnetic and paramagnetic parts are deducted, and the results are shown in Fig. 2 (c). Compared with others, obviously increased saturation magnetization $\left(M_{s}\right)$ while decreased coercive field $\left(H_{c}\right)$ (inset of Fig. 2(c)) are observed for the $62 \mathrm{~nm}$ BFO particles, which further approves the distinct origin of its ferromagnetism. We would like to mention that, all the results shown in this paper can be fully repeated for different batches of our sample.

Electron energy loss spectroscopy (EELS) inspection may provide the clue for the abnormal magnetism of $62 \mathrm{~nm}$ particles. As shown in Fig. 3 (a) and in supplementary Fig. S6, two typical peaks of oxygen $K$-edge located at about $531 \mathrm{eV}$ and $540 \mathrm{eV}$ are observed for all the particles. While interestingly, obvious furcation of the two peaks are observed only for the $62 \mathrm{~nm}$ BFO particles, indicating the changed bonding information of oxygen ${ }^{25}$ and implying that a structural anomaly of a so-far unknown type is present for the particle. Since it cannot be observed in XRD measurement and refinement, we argue that the structural anomaly is merely an enhanced distortion without symmetry change, such as the increased rotation distortion of $\mathrm{FeO}_{6}$ octahedron around the [111] direction, which can cause the strengthening of Dzyaloshinskii-Moriya (DM) interaction and the suppression of the spiral spin structure ${ }^{26-28}$, and thus increase the ferromagnetism of the $62 \mathrm{~nm}$ BFO particles. Theoretical results further confirm this inference. First, dependence of spontaneous magnetization on the rotation angle of $\mathrm{FeO}_{6}$ octahedron around [111] direction is analyzed by first principles calculation ${ }^{29}$. We fix the positions of cations and change the coordinates of oxygen ions to obtain different rotation of $\mathrm{FeO}_{6}$ octahedron, and then calculate the

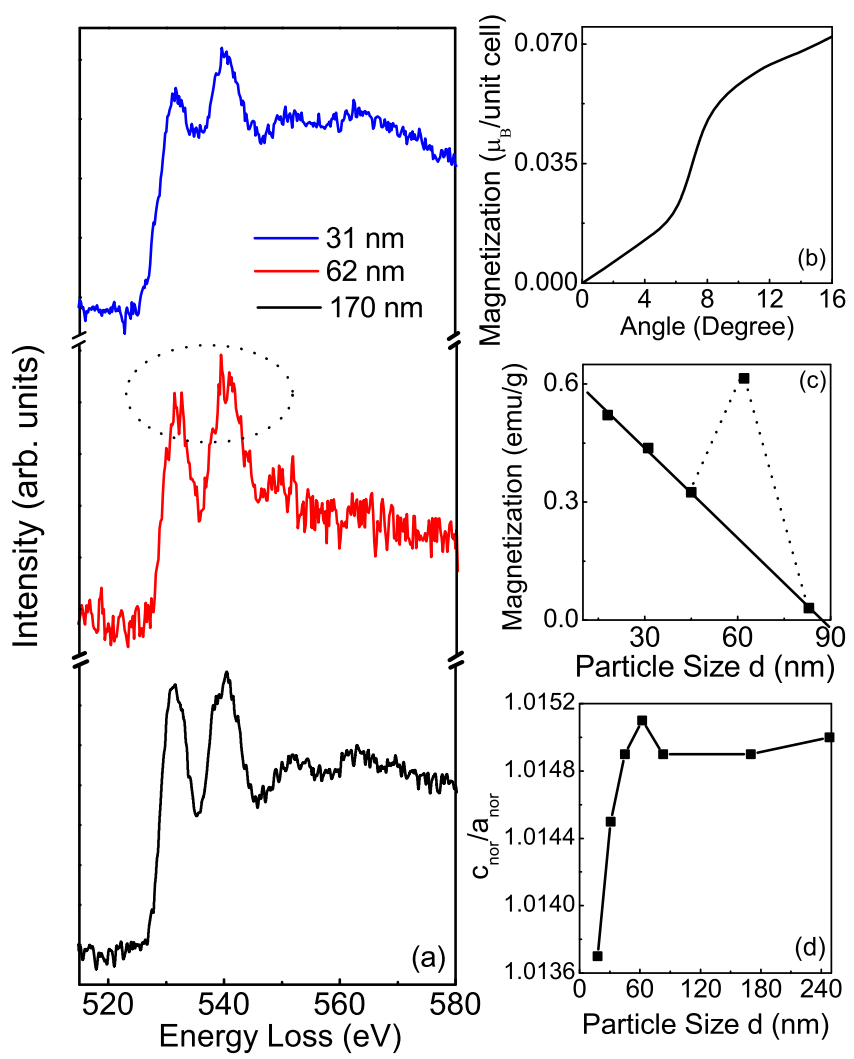

Figure 3 (a) Electron energy loss spectroscopy of oxygen $K$ edges for $31 \mathrm{~nm}, 62 \mathrm{~nm}$ and $170 \mathrm{~nm}$ BFO particles. (b) Dependence of magnetization per unit cell on the rotation angle of $\mathrm{FeO}_{6}$ octahedron. (c) Size-dependent spontaneous magnetization. (d) Size-dependent $\frac{c_{\text {nor }}}{a_{n o r}}$. 
spontaneous magnetization under different rotation angles. Fig. 3 (b) shows that the magnetization per unit cell increases with the increased angle, indicating that local ferromagnetism of BFO can be increased by the increased rotation distortion of $\mathrm{FeO}_{6}$ octahedron through the strengthened DM interaction. Second, based on the spherical particle model, the macroscopic magnetization are calculated as a function of particle size according to the method given in our former paper $^{29}$. For the $62 \mathrm{~nm}$ BFO particles, the ferromagnetism of DM type is calculated by first principles calculation as mentioned above supposing the rotation angle increases by $2^{\circ}$ from $12.8^{\circ}$ - that reported for bulk BFO ceramics at room temperature ${ }^{30}$. As shown in Fig. 3 (c), obviously increased magnetization are also obtained for the $62 \mathrm{~nm}$ BFO particles, which is in reasonable agreement with the experimental behavior given in the inset of Fig. 2(c). The relatively larger calculated magnetization for all the particles compared with our experiments probably comes from the deviation of the rotation angle, the randomly oriented grains and the irregular particle shape. In addition, the enhanced DM interaction can also induce improved ferroelectric property ${ }^{31}$, which is always reflected by increased $\frac{c_{\text {nor }}}{a_{\text {nor }}}$ value, where $a_{\text {nor }}$ and $c_{\text {nor }}$ are the lattice parameters of tetragonal structure calculated from $a_{\text {nor }}=\frac{a}{\sqrt{2}}$, and $c_{\text {nor }}=\frac{c}{\sqrt{2}}^{32}$. Figure $3(d)$ depicts the variation of $\frac{c_{\text {nor }}}{a_{\text {nor }}}$ with particle size. Similar to the result given by Selbach et al. ${ }^{12}$, with the decrease of particle size, the $\frac{c_{\text {nor }}}{a_{\text {nor }}}$ value first decreases slowly and then abruptly with $30 \mathrm{~nm}$ as an inflexion point. However, an abnormal point corresponding to an obviously increased $\frac{c_{\text {nor }}}{a_{\text {nor }}}$ is observed for the $62 \mathrm{~nm}$ BFO particles, indicating its ferroelectric property is improved probably by the enhanced DM interaction through magnetoelecric coupling. Taken together, a structural anomaly probably corresponding to the increased rotation distortion of $\mathrm{FeO}_{6}$ octahedron is present when the particle size approaches the period of the spiral spin structure. This structural anomaly could cause an increased DM interaction, and in turn a suppressed spiral spin structure, which finally result in an increased ferromagnetism of BFO particles with size around $62 \mathrm{~nm}$. While BFO nanoparticles with other sizes can be modeled by a superposition of an antiferromagnetic core and a ferromagnetic surface, as a result, the ferromagnetism contributed by the uncompensated spin at grain surface increases as particle size decreases.

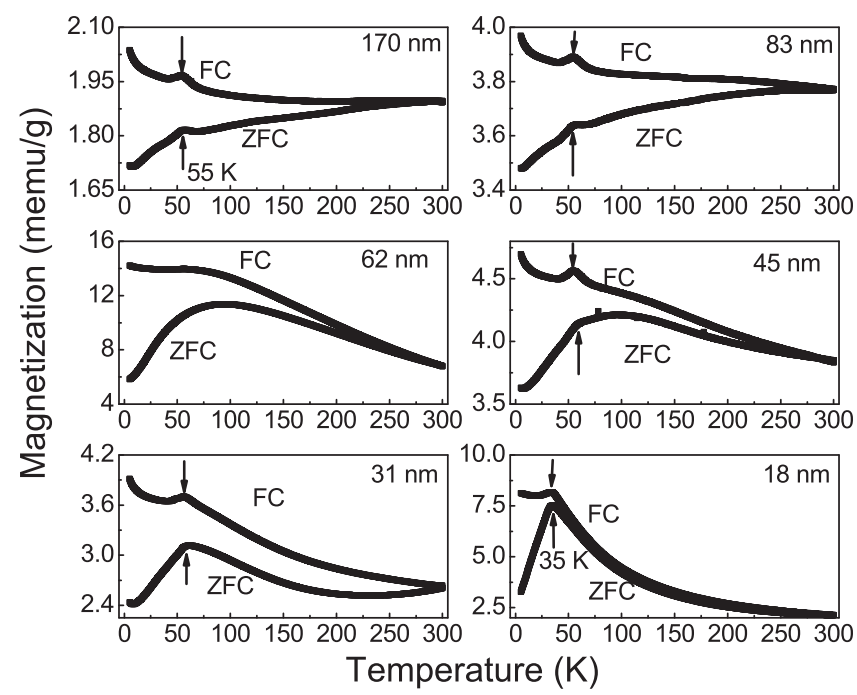

Figure $4 \mid$ Magnetization as a function of temperature measured under ZFC and 200 Oe FC conditions for BFO nanoparticles with various sizes.
Besides the room temperature magnetic property, the particularity of $62 \mathrm{~nm}$ particles can also be gleaned from temperature dependent magnetization studies. Figure 4 shows the magnetization of our BFO nanoparticles as a function of temperature, measured at 200 Oe after zero-field cooling (ZFC) and also with 200 Oe field cooling (FC). There are three features worth mentioning: (1) The splitting in the ZFC and FC magnetization curves are observed for all the BFO nanoparticles. (2) With temperature decreasing from $300 \mathrm{~K}$ to $10 \mathrm{~K}$, the ZFC magnetization value $\left(M_{Z F C}\right)$ monotonically decreases for the $170 \mathrm{~nm}$ and $83 \mathrm{~nm}$ BFO particles, while it first increases and then decreases, as a result, a broad peak appears in the ZFC curves for the BFO particles with size less than $62 \mathrm{~nm}$. (3) Except for the absence in $62 \mathrm{~nm}$ particles, a sharp cusp (shown in Figure 4 by arrows) is observed in the ZFC and FC curves for other BFO nanoparticles. Here we try to explain the above features one by one.

1) The splitting of the ZFC and FC curves usually appears in the co-existent system of antiferromagnetic and ferromagnetic phases ${ }^{33}$, which coincides with the core-shell structure proposed above, that is, our BFO nanoparticles are composed of an antiferromagnetic core and a ferromagnetic surface. Though the spiral-modulated spin structure is probably suppressed for the $62 \mathrm{~nm}$ BFO particles by the increased rotation distortion of $\mathrm{FeO}_{6}$ octahedron, local antiferromagnetic interaction still exists in the particle. 2) The ferromagnetic behavior of the BFO particles with size less than $62 \mathrm{~nm}$ is responsible for the increased $M_{Z F C}$ with decreasing temperature, and the presence of the broad peak in ZFC curves can be attributed to the magnetic blocking mechanism induced by the competition between thermal energy and magnetic anisotropy energy. 3) The sharp cusp presented around $55 \mathrm{~K}\left(T_{\text {cusp }}\right)$ in ZFC and FC curves has been attributed to superparamagnetic relaxation process ${ }^{19}$ and the spin glass behavior ${ }^{20}$. If the cusp represents a blocking behavior associated with superparamagnetic relaxation processes of isolated magnetic nanoparticles, one would expect a stronger dependence of $T_{\text {cusp }}$ on particle size ${ }^{34}$. However, as shown in Fig. $4, T_{\text {cusp }}$ is about $55 \mathrm{~K}$ for most of our BFO particles with different sizes $(31 \mathrm{~nm}$, $45 \mathrm{~nm}, 83 \mathrm{~nm}$ and $170 \mathrm{~nm}$ ), and even for $248 \mathrm{~nm}$ particles (Supplementary Fig. S7) and bulk BFO ceramics ${ }^{15}$. On the other hand, Vijayanand et al. proved that the above-mentioned cusp is not associated with any spin glass or phase transition by a series of ZFC and FC magnetization measurement, and they believe that it corresponds to some changes associated with domain structure ${ }^{21}$. Here, we consider that the sharp cusp of our BFO nanoparticles may be related to the magnetic domain pinning effect, which is well known in magnetic materials ${ }^{35}$. However, why the cusp could not be observed in the $62 \mathrm{~nm}$ BFO particles? In order to answer this

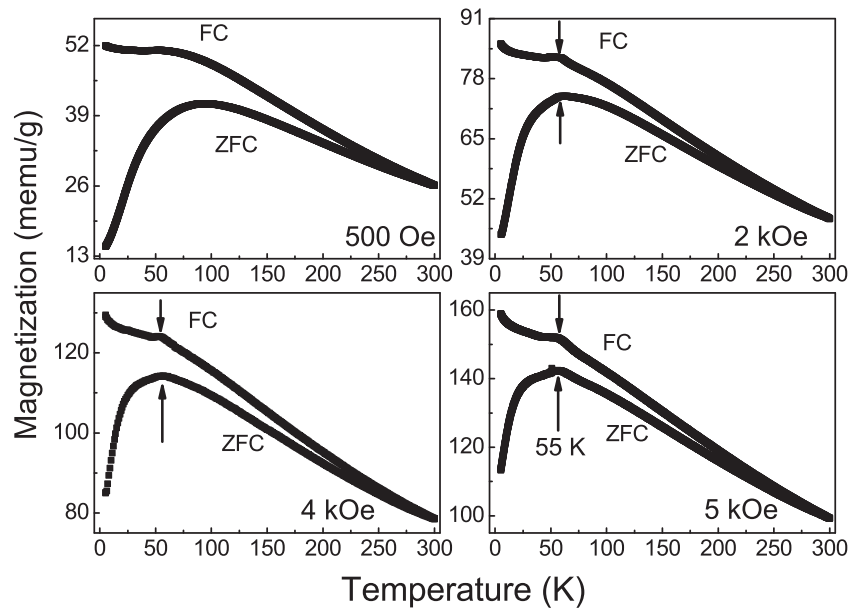

Figure 5| ZFC and FC magnetization curves measured with various magnetic fields for the $62 \mathrm{~nm}$ BFO particles. 
question, a series of ZFC and FC curves are measured under various magnetic fields. As shown in Fig. 5, the cusp at about $55 \mathrm{~K}$ can also be observed for the $62 \mathrm{~nm}$ BFO particles when the applied magnetic field is larger than $2 \mathrm{kOe}$, where most of the ferromagnetic domains are switched to the direction of the applied magnetic field. Therefore, the cusp at about $55 \mathrm{~K}$, which can be observed in BFO nanoparticles, ceramics, and single crystals, is related to the antiferromagnetic domain pinning effect. The origin of the domain pining is usually ascribed to the non-uniformly distributed defects, which affects the movement of domain walls. As shown in Fig. 4, although the shape of the cusp is difficult to be distinguished from the ZFC curves because of the superimposition of the broad peak originated from magnetic blocking, it can be clearly seen from the FC curves that the cusp height is almost the same for the BFO nanoparticles with various sizes, indicating their similar pining behavior. As for the $18 \mathrm{~nm} \mathrm{BFO}$ particles, lower $T_{\text {cusp }}$ of about $35 \mathrm{~K}$ is obtained probably because of the possible interparticle interactions.

Raman spectra are performed and shown in Fig. 6(a) since it can give some indications of spin-phonon coupling ${ }^{36}$. Similar to the literatures $^{15,37}$, peaks at about $136 \mathrm{~cm}^{-1}$ (peak A), $168 \mathrm{~cm}^{-1}$ (peak B) and $218 \mathrm{~cm}^{-1}$ (peak $\mathrm{C}$ ) are observed, which can be attributed to the manifestation of $A_{1}$ modes. Since the suppressed intensity of peak $A$ implies the enhanced coupling of magnetic, ferroelectric and/or structural order parameters ${ }^{15}$, we fit the Raman spectra using WiRE2.0 software and estimate the suppressed degree of peak A by $I_{A} / I_{B}$, where $I_{A}$ and $I_{B}$ represent the integral intensities of peak $A$ and peak $B$, respectively. It is seen from Fig. 6(a) that obviously decreased $\mathrm{I}_{\mathrm{A}} / \mathrm{I}_{\mathrm{B}}$ values are observed for the BFO particles smaller than $62 \mathrm{~nm}$, which coincides well with their enhanced ferromagnetic properties. Furthermore, the smallest $\mathrm{I}_{\mathrm{A}} / \mathrm{I}_{\mathrm{B}}$ value of the $62 \mathrm{~nm}$ BFO particles probably indicates the increased magnetoelectric coupling in this special particle.
To gain insight into the magnetoelectric coupling of our BFO nanoparticles, magnetodielectric (MD) effect, i.e the variation in dielectric constant $\varepsilon$ under an applied bias magnetic field $\left(\mathrm{MD}=\frac{\varepsilon_{\mathrm{r}}(\mathrm{H})-\varepsilon_{\mathrm{r}}(0)}{\varepsilon_{\mathrm{r}}(0)}\right.$, where $\varepsilon_{\mathrm{r}}(\mathrm{H})$ and $\varepsilon_{\mathrm{r}}(0)$ are the dielectric constant measured under and without applied magnetic field, respectively), is investigated. It is said that the MD effect can be attributed by not only the intrinsic coupling of spontaneous polarization and magnetization, but also the extrinsic coupling contributed by a combination of magnetoresistance and Maxwell-Wagner effect ${ }^{38,39}$, and the MD effect measured below $700 \mathrm{kHz}$ cannot truly reflect the intrinsic magnetoelectric coupling ${ }^{38-40}$. Thus, the MD effect present in Fig. 6 (b-d) and Fig. S8 (Supplementary information) of our BFO nanoparticles are measured at $1 \mathrm{MHz}$ for the temperature range from $4 \mathrm{~K}$ to $310 \mathrm{~K}$. In fact, as shown in the inset of Fig. 6 (b), almost frequency independent dielectric constant is observed for the $62 \mathrm{~nm} \mathrm{BFO}$ particles when the frequency range from $10^{4.5}$ to $10^{6} \mathrm{~Hz}$, indicating that the MD effect of our BFO nanoparticles are mainly come from the intrinsic coupling between spontaneous polarization and magnetization. The MD effect over the whole temperature range is clearly demonstrated for the $62 \mathrm{~nm}, 31 \mathrm{~nm}$ and $18 \mathrm{~nm}$ BFO particles (Fig. 6 (b) and (c)), which is much obvious than that observed in other particles (Supplementary Fig. S8). More clearly, as shown in Fig. 6 (d), larger MD values are obtained in the temperature range 4$310 \mathrm{~K}$ for the $62 \mathrm{~nm} \mathrm{BFO}$ particles, which is much larger than that of the nanoparticles with other size and that reported for some doped BFO materials ${ }^{40,41}$. The greater MD effect of $62 \mathrm{~nm}$ BFO particles corresponds to its improved ferromagnetic, ferroelectric and magnetoelectric coupling properties probably related with the enhanced rotation distortion of $\mathrm{FeO}_{6}$ octahedron. In addition, the dielectric anomaly observed at around $250 \mathrm{~K}$ for our BFO nanoparticles (shown in Fig. 6 (b) and (c) by arrows, and also in supplementary Fig. S9) could be ascribed to surface effect according to the recently
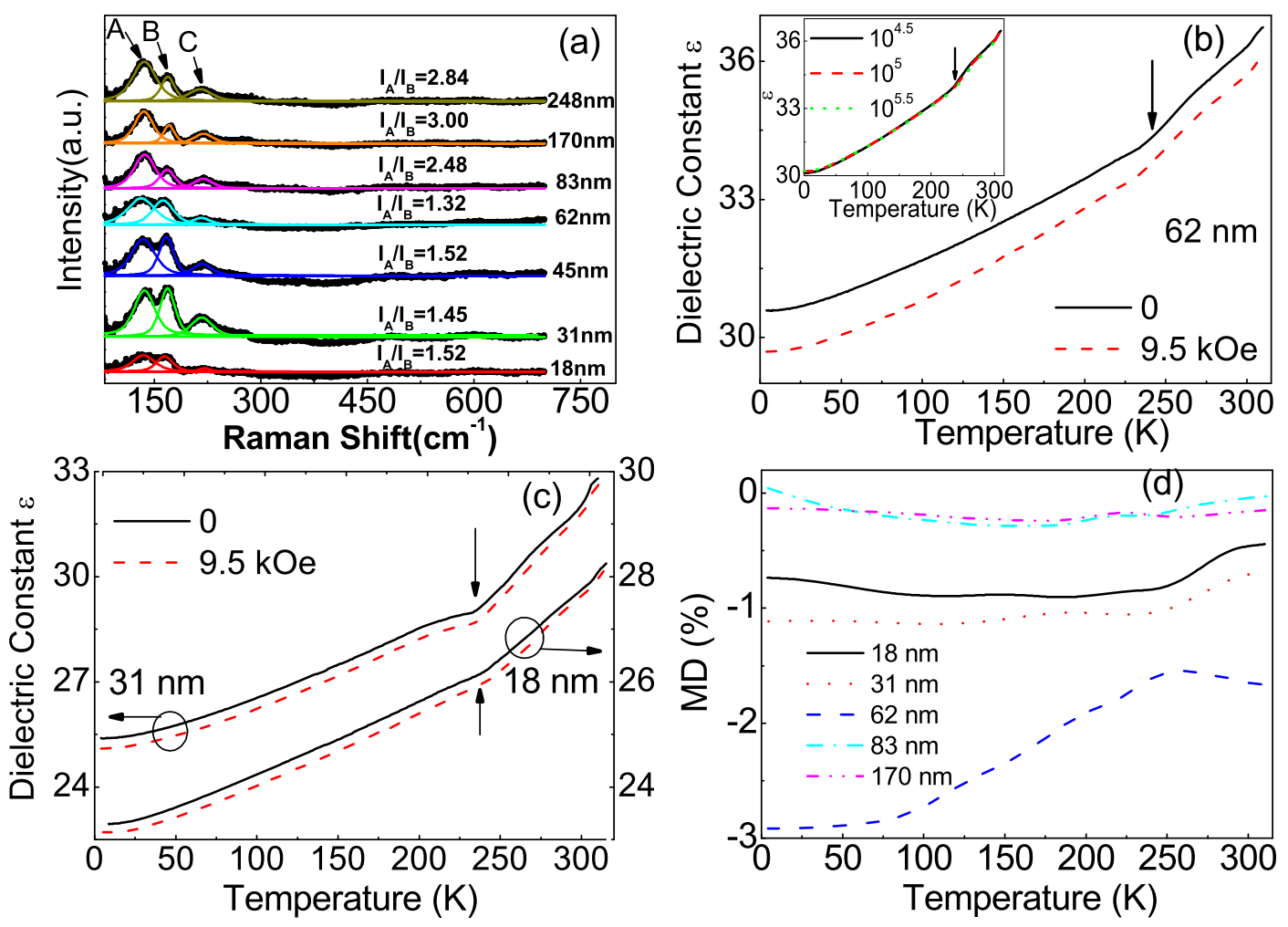

Figure $6 \mid$ (a) Raman spectra of BFO nanoparticles with various sizes, in which the black lines and color lines correspond to the measured and fitted results, respectively. Temperature dependent dielectric constant under zero and $9.5 \mathrm{kOe}$ magnetic field for the (b) $62 \mathrm{~nm}$, (c) $31 \mathrm{~nm}$ and $18 \mathrm{~nm}$ particles. The inset in (b) gives the temperature dependent dielectric constant of the $62 \mathrm{~nm}$ BFO particles measured at different frequencies. (d) Temperature dependent MD effect for the BFO nanoparticles with various sizes. 
study reported by Jarrier et $a .^{42}$ since no magnetic anomaly is observed in neutron data for BFO single crystal below room temperature ${ }^{43,44}$.

\section{Discussion}

Up to now, most studies on the size effect of ferroelectric and magnetic materials have revealed that their properties usually vary monotonically with decreasing size. For example, the ferromagnetism contributed by the uncompensated spins at grain surface is enhanced with decreasing sample size in antiferromagnetic materials, which is also observed in our BFO nanoparticles. However, we find some unexpected changes of the properties when the particle size is around $62 \mathrm{~nm}$, such as the obviously increased ferromagnetism and magnetoelectric coupling, which are related with a structural anomaly probably being an enhanced rotation distortion of $\mathrm{FeO}_{6}$ octahedron. This report reveals for the first time that, special distortion of lattice and magnetic structure can be induced when the sample size is close to the spiral period, which can further lead to extraordinary macroscopic properties. On the one hand, the results provide novel approaches to improve the ferromagnetism and magnetoelectric coupling of BFO, which is important for its applications since the antiferromagnetic nature and poor coupling between electric and magnetic properties are the primary weakness of BFO as room temperature multiferroics. On the other hand, our work not only highlights the important regulatory role of the spiral spin period on the properties of BFO but also calls forth more attention on the size effect of materials with periodically modulated spin structure. Further studies both theoretical and experimental are urgently demanded in order to understand what really happens when the sample size approaches the period of the spin-structure.

In summary, BFO nanoparticles with various sizes are prepared by a sol-gel method. For the particles with size close to the spiral spin period of BFO, a structural anomaly arises, which improves the multiferroic and magnetoelectric coupling properties of the material. The present work highlights the role of the spiral spin structure on the modulation of microscopic properties, and opens a new window for the application of BFO.

\section{Methods}

Sample preparation. BFO nanoparticles with size from 18 to $248 \mathrm{~nm}$ were prepared by a sol-gel method. The precursor solution was prepared by dissolving $10 \mathrm{mmol}$ $\mathrm{Bi}\left(\mathrm{NO}_{3}\right)_{3} \cdot 5 \mathrm{H}_{2} \mathrm{O}$ and $10 \mathrm{mmol} \mathrm{Fe}\left(\mathrm{NO}_{3}\right)_{3} \cdot 9 \mathrm{H}_{2} \mathrm{O}$ in distilled water with the addition of dilute nitric acid, wherein tartaric acid as organic complex was added with 1:1 molar ratio to the cations. The mixture was stirred for several hours, and then evaporated and dried at $140^{\circ} \mathrm{C}$ for $15 \mathrm{~h}$ to obtain the xerogel powder. Finally, the xerogel were calcined at temperatures varied from 425 to $575^{\circ} \mathrm{C}$ for $2 \mathrm{~h}$ to obtain BFO nanoparticles with various sizes.

Structure characterization. X-ray diffraction with $\mathrm{Cu} \mathrm{k} \alpha$ radiation was used for analyzing the structure of the BFO nanoparticles. Scanning electron microscopy, transmission electron microscopy (FEI Tecnai F20) were used for analyzing the size and microstructure of the particles. Raman spectra were performed on micro-Raman spectrometer (Horiba Jobin Yvon HR800). The electron energy loss spectroscopy (EELS) were conducted on a FEI TITAN 80-300 field emission TEM equipped with a Gatan imaging filter system (model: Tridiem 863). The microscope was operated at an accelerating voltage of $300 \mathrm{kV}$, and the spectra were acquired in diffraction mode.

Magnetic and dielectric measurements. The magnetic properties were measured with a commercial magnetic property measurement system. For the measurement of dielectric properties, the nanoparticles were pressed into a tetragonal shape $(3 \times 3 \times$ $0.3 \mathrm{~mm}^{3}$ ) at $10 \mathrm{MPa}$, then, silver paste was painted on both sides of the sample as a capacitance. The dielectric properties as well as the MD effect were investigated using HP4194A impedance analyzer with home-built sample holder coupled with a helium closed cycle refrigerator (temperature range from $4 \mathrm{~K}$ to $310 \mathrm{~K}$ ) and applied magnetic field.

First-principles calculation. Our first-principles calculations were performed using the projector-augmented wave (PAW) method within the local spin-density approximation plus the on-site repulsion (LSDA + U), as implemented in the Vienna $\mathrm{ab}$ initio simulation package (VASP). We used $\mathrm{U}=4 \mathrm{eV}$ on Fe $3 \mathrm{~d}$ states and a planewave cutoff energy of $500 \mathrm{eV}$. The calculations were performed with a $5 \times 5 \times 5 \mathrm{k}$ point sampling. We switched on the spin-orbit coupling (SOC) and employed the convergence threshold of $10^{-7} \mathrm{eV}$ due to the small value of SOC. For the calculations of spontaneous magnetization, we set the initial spins collinear, and allow the spins relax freely within the self-consistency cycle to induce canted magnetic moment.

1. Schmid, H. Multiferroic Magnetoelectrics. Ferroelectrics 162, 317-338 (1994)

2. Eerenstein, W., Mathur, N. D. \& Scott, J. F. Multiferroic and magnetoelectric materials. Nature 442, 759-765 (2006).

3. Fiebig, M. Revival of the magnetoelectric effect. J. Phys. D-Appl. Phys. 38, R123-R152 (2005)

4. Martin, L. W., Chu, Y. H. \& Ramesh, R. Advances in the growth and characterization of magnetic, ferroelectric, and multiferroic oxide thin films. Mater. Sci. Eng. R 68, 89-133 (2010).

5. Spaldin, N. A. \& Fiebig, M. The renaissance of magnetoelectric multiferroics. Science 309, 391-392 (2005).

6. Lottermoser, T. et al. Magnetic phase control by an electric field. Nature 430 541-544 (2004)

7. Fischer, P., Polomska, M., Sosnowska, I. \& Szymanski, M. Temperaturedependence of the crystal and magnetic-structures of $\mathrm{BiFeO}_{3}$. J. Phys. C-Solid State Phys. 13, 1931-1940 (1980).

8. Ederer, C. \& Spaldin, N. A. Influence of strain and oxygen vacancies on the magnetoelectric properties of multiferroic bismuth ferrite. Phys. Rev. B 71, 224103 (2005).

9. Sosnowska, I., Peterlinneumaier, T. \& Steichele, E. Spiral magnetic-ordering in bismuth ferrite. J. Phys. C-Solid State Phys. 15, 4835-4846 (1982).

10. Catalan, G. \& Scott, J. F. Physics and Applications of Bismuth Ferrite. Adv. Mater 21, 2463-2485 (2009).

11. Hong, S. et al. Large Resistive Switching in Ferroelectric $\mathrm{BiFeO}_{3} \mathrm{Nano}-\mathrm{Island}$ Based Switchable Diodes. Adv. Mater. 25, 2339-2343 (2013).

12. Selbach, S. M., Tybell, T., Einarsrud, M. A. \& Grande, T. Size-dependent properties of multiferroic $\mathrm{BiFeO}_{3}$ nanoparticles. Chem. Mater. 19, 6478-6484 (2007).

13. Chen, P. et al. Size-Dependent Infrared Phonon Modes and Ferroelectric Phase Transition in $\mathrm{BiFeO}_{3}$ Nanoparticles. Nano Lett. 10, 4526-4532 (2010).

14. Reddy, V. A., Pathak, N. P. \& Nath, R. Particle size dependent magnetic properties and phase transitions in multiferroic $\mathrm{BiFeO}_{3}$ nano-particles. J. Alloy. Compd. 543, 206-212 (2012)

15. Park, T. J., Papaefthymiou, G. C., Viescas, A. J., Moodenbaugh, A. R. \& Wong, S. S. Size-dependent magnetic properties of single-crystalline multiferroic $\mathrm{BiFeO}_{3}$ nanoparticles. Nano Lett. 7, 766-772 (2007).

16. Gao, F. et al. Preparation and photoabsorption characterization of $\mathrm{BiFeO}_{3}$ nanowires. Appl. Phys. Lett. 89, 102506 (2006).

17. Mazumder, R. et al. Particle size dependence of magnetization and phase transition near T-N in multiferroic $\mathrm{BiFeO}_{3}$. J. Appl. Phys. 100, 033908 (2006).

18. Richardson, J. T., Yiagas, D. I., Turk, B., Forster, K. \& Twigg, M. V. Origin of superparamagnetism in nickel-oxide. J. Appl. Phys. 70, 6977-6982 (1991).

19. Singh, M. K., Prellier, W., Singh, M. P., Katiyar, R. S. \& Scott, J. F. Spin-glass transition in single-crystal $\mathrm{BiFeO}_{3}$. Phys. Rev. B 77, 144403 (2008).

20. Singh, M. K., Katiyar, R. S., Prellier, W. \& Scott, J. F. The Almeida-Thouless line in $\mathrm{BiFeO}_{3}$ : is bismuth ferrite a mean field spin glass? J. Phys.-Condens. Matter 21, 042202 (2009).

21. Vijayanand, S., Mahajan, M. B., Potdar, H. S. \& Joy, P. A. Magnetic characteristics of nanocrystalline multiferroic $\mathrm{BiFeO}_{3}$ at low temperatures. Phys. Rev. B 80, 064423 (2009).

22. Jaiswal, A. et al. Effect of Reduced Particle Size on the Magnetic Properties of Chemically Synthesized $\mathrm{BiFeO}_{3}$ Nanocrystals. J. Phys. Chem. C 114, 2108-2115 (2010).

23. Huang, F. Z. et al. Thickness-dependent structural and magnetic properties of $\mathrm{BiFeO}_{3}$ films prepared by metal organic decomposition method. Appl. Phys. Lett. 97, 222901 (2010).

24. Joy, P. A. \& Date, S. K. Comparison of the zero-field-cooled magnetization behavior of some ferromagnetic and ferrimagnetic systems. J. Magn. Magn. Mater. 218, 229-237 (2000).

25. Garvie, L. A. J. Can electron energy-loss spectroscopy (EELS) be used to quantify hydrogen in minerals from the O K edge? Am. Mineral. 95, 92-97 (2010).

26. Ederer, C. \& Spaldin, N. A. Weak ferromagnetism and magnetoelectric coupling in bismuth ferrite. Phys. Rev. B 71, 060401 (2005).

27. Huang, F. Z. et al. Effect of Nd dopant on magnetic and electric properties of $\mathrm{BiFeO}_{3}$ thin films prepared by metal organic deposition method. Appl. Phys. Lett. 89, 242914 (2006).

28. Ramazanoglu, M. et al. Local Weak Ferromagnetism in Single-Crystalline Ferroelectric $\mathrm{BiFeO}_{3}$. Phys. Rev. Lett. 107, 207206 (2011).

29. Zhang, J. T. et al. Origin of magnetic anisotropy and spiral spin order in multiferroic $\mathrm{BiFeO}_{3}$. Appl. Phys. Lett. 100, 242413 (2012).

30. Palewicz, A., Przeniosło, R., Sosnowska, I. \& Hewat, A. W. Atomic displacements in $\mathrm{BiFeO}_{3}$ as a function of temperature: neutron diffraction study. Acta. Cryst. B63, 537-544 (2007).

31. Matsuda, M. et al. Magnetic Dispersion and Anisotropy in Multiferroic $\mathrm{BiFeO}_{3}$. Phys. Rev. Lett. 109, 067205 (2012).

32. Chakrabarti, K. et al. Enhanced magnetic and dielectric properties of Eu and Co co-doped $\mathrm{BiFeO}_{3}$ nanoparticles. Appl. Phys. Lett. 101, 042401 (2012). 
33. Huang, F. Z. et al. Multiferroic properties of Co and Nd co-substituted $\mathrm{Bi}_{5} \mathrm{Ti}_{3} \mathrm{FeO}_{15}$ thin films. Thin Solid Films 520, 6489-6492 (2012).

34. Mamiya, H., Nakatani, I. \& Furubayashi, T. Blocking and freezing of magnetic moments for iron nitride fine particle systems. Phys. Rev. Lett. 80, 177-180 (1998).

35. Sankar, C. R. \& Joy, P. A. Magnetic properties of the self-doped lanthanum manganites $\mathrm{La}_{1-\mathrm{x}} \mathrm{MnO}_{3}$. Phys. Rev. B 72, 024405 (2005).

36. Haumont, R., Kreisel, J., Bouvier, P. \& Hippert, F. Phonon anomalies and the ferroelectric phase transition in multiferroic $\mathrm{BiFeO}_{3}$. Phys. Rev. B 73, 132101 (2006).

37. Singh, M. K., Ryu, S. \& Jang, H. M. Polarized Raman scattering of multiferroic $\mathrm{BiFeO}_{3}$ thin films with pseudo-tetragonal symmetry. Phys. Rev. B 72, 132101 (2005).

38. Kamba, S. et al. Infrared and terahertz studies of polar phonons and magnetodielectric effect in multiferroic $\mathrm{BiFeO}_{3}$ ceramics. Phys. Rev. B 75, 024403 (2007).

39. Catalan, G. Magnetocapacitance without magnetoelectric coupling. Appl. Phys. Lett. 88, 102902 (2006).

40. Jang, H. M., Park, J. H., Ryu, S. W. \& Shannigrahi, S. R. Magnetoelectric coupling susceptibility from magnetodielectric effect. Appl. Phys. Lett. 93, 252904 (2008).

41. Wang, D. H., Goh, W. C., Ning, M. \& Ong, C. K. Effect of Ba doping on magnetic, ferroelectric, and magnetoelectric properties in mutiferroic $\mathrm{BiFeO}_{3}$ at room temperature. Appl. Phys. Lett. 88, 212907 (2006).

42. Jarrier, R. et al. Surface phase transitions in $\mathrm{BiFeO}_{3}$ below room temperature. Phys. Rev. B 85, 184104 (2012).

43. Ramazanoglu, M. et al. Temperature-dependent properties of the magnetic order in single-crystal $\mathrm{BiFeO}_{3}$. Phys. Rev. B 83, 174434 (2011)

44. Herrero-Albillos, J. et al. Neutron diffraction study of the $\mathrm{BiFeO}_{3}$ spin cycloid at low temperature. J. Phys. Condens. Matter 22, 256001 (2010).

\section{Acknowledgements}

This work was supported by the 973 Project of MOST (No. 2009CB929501), National Science Foundation of China (Nos. 51002075, 51225201, 61271078, and 51102133), the Priority Academic Program Development of Jiangsu Higher Education Institutions (PAPD) and the Fundamental Research Funds for the Central Universities.

\section{Author contributions}

F.Z.H. and X.M.L. designed the experiments and wrote the paper. F.Z.H. and Z.J.W. prepared the sample and performed the structural measurements. F.Z.H. and K.L.M. contributed to the measurements of dielectric and MD effect. R.X.T., T.T.X., J.H. and C.Y. performed the magnetic measurements. J.T.Z. conducted the calculations. F.Z.H., X.M.L., W.W.L., J.T.Z. and J.S.Z. analyzed and discussed the data. All authors contributed through scientific discussions.

\section{Additional information}

Supplementary information accompanies this paper at http://www.nature.com/ scientificreports

Competing financial interests: The authors declare no competing financial interests. How to cite this article: Huang, F.Z. et al. Peculiar magnetism of $\mathrm{BiFeO}_{3}$ nanoparticles with size approaching the period of the spiral spin structure. Sci. Rep. 3, 2907; DOI:10.1038/ srep02907 (2013)

\section{(c) (i) (2) (2) This work is licensed under a Creative Commons Attribution-}

NonCommercial-ShareAlike 3.0 Unported license. To view a copy of this license, visit http://creativecommons.org/licenses/by-nc-sa/3.0 